subgroups according to their infectious status and having clinical signs or not. CRP values were compared between neonates infected and not infected. Receiver operating characteristic (ROC) curves were constructed separately in symptomatic and asymptomatic groups. The sensitivity, specificity, positive predict value, negative predict value, likelihood ratios and posttest probability were determined using different cut-off values. Results 24,344 infants with 28,830 CRP results were included. Early onset infection was confirmed in 75 (0.31\%) cases, 68 of whom had EOS and 7 had local infections (urinary tract or eyes). Discrimination improved after 8 hours of birth in asymptomatic neonates (the area under the ROC curve (AUC) was 0.49 at $<8$ hours and 0.79 at $\geq 8$ hours), and 24 hours of birth in symptomatic neonates (the AUC was 0.65 at $<24$ hours and 0.75 at $\geq 24$ hours). High CRP values were less informative in symptomatic neonates than asymptomatic neonates (eg. the likelihood ratio for CRP>10 mg/L was 2.2 in symptomatic and 5 in asymptomatic neonates). Using cut off value of $10 \mathrm{mg} / \mathrm{L}$ at $\geq 24$ hours, sensitivity was $72 \%$ in symptomatic neonates and $83 \%$ in asymptomatic neonates, and specificity was $69 \%$ and $86 \%$ respectively. Positive predict values were very low $(0.3-6 \%)$.

Conclusions Diagnostic performance of CRP was poor within 24 hours of birth. However, in asymptomatic neonates, high CRP in 8-24 hours was informative of infection. Because of the low positive predict value, attention should be paid to overtreatment when using CRP for decision making.

\section{CONTEMPORARY TRENDS IN GLOBAL MORTALITY OF NEONATAL SEPSIS: A SYSTEMATIC REVIEW AND META- ANALYSIS}

Wen Li Lee, Ming Ying Gan, Bei Jun Yap, Tammie Seethor, Bobby Tan, Shu-Ling Chong. Singapore and United States of America

\subsection{6/bmjpo-2021-RCPCH.212}

Background Sepsis causes death and morbidity in young infants. Globally, an estimated 1.3 - 3.9 million young infants experience sepsis and 400,000 - 700,000 die from sepsisrelated conditions annually. Even though there have been significant progress over the past twenty years in reducing young infant mortality, sepsis currently accounts for up to $15 \%$ of all young infant deaths. A thorough understanding of young infant sepsis can inform strategies that span prevention, diagnosis and intervention for young infant sepsis.

Objectives We aimed to perform a systematic review and meta-analysis to investigate the case fatality rates (CFRs) among young infants less than 90 days with sepsis globally.

Methods We used the Preferred Reporting Items for Systematic Reviews and Meta-analysis (PRISMA) 2009 guidelines. We searched PubMed, Cochrane Central, Embase and Web of Science for randomized clinical trials and observational studies in English language, published between 2010 to 2019. Studies involving young infants less than 90 days old with sepsis and reported CFRs were included. We obtained pooled CFRs estimates using the random effects model. Additional stratifications by gestation, birth weight, onset of sepsis (early onset was defined as $<72$ hours), source of sepsis and gross national income were also performed. Risk of bias was assessed using the Cochrane risk-of-bias tool for randomized controlled trials, and the Newcastle-Ottawa Scale for all observational studies.
Results Among 6314 articles screened, 240 studies with a total of 437,796 patients met the inclusion criteria and were included in our analysis. 99 came from high income countries, 44 from upper middle income countries, 82 from lower middle income countries, 6 from low income countries and 9 were conducted in multiple countries. Overall, the pooled CFR was 0.18 (95\% CI, 0.17-0.19). The CFR was the highest in low income countries $(0.25$ [95\% CI, 0.07-0.43]), followed by lower middle (0.24 [95\% CI, 0.21-0.26]), upper middle $(0.21$ [95\% CI, 0.18-0.24]) and lastly high income countries (0.12 [95\% CI, 0.11-0.13]).

Other factors associated with higher CFRs included prematurity $(0.23$ [95\% CI, 0.19-0.26] vs term CFR 0.10 [95\% CI, $0.08-0.13])$, low birth weight $(0.21$ [95\% CI, 0.19-0.24] vs normal birth weight 0.19 [95\% CI, 0.18-0.20]), early onset sepsis $(0.20$ [95\% CI, 0.17-0.24] vs combined (0.16 [95\% CI, $0.14-0.18])$ and hospital acquired infection (0.23 [95\% CI, $0.17-0.30]$ vs community acquired infections 0.21 [95\% CI, $0.10-0.33])$. Time trend analysis showed higher CFRs in the low income countries than the middle and high income countries. A decreasing trend in CFRs over time was observed in high and upper middle income countries, as compared to an increasing trend in lower middle and low income countries.

Conclusions While we saw a declining trend of young infant sepsis CFRs among high and upper middle income countries across the years, the increasing trend amongst lower middle and low income countries highlights a disparity in infant sepsis outcomes based on resource availability. We highlight specific vulnerable patient populations that should be further studied in order to reduce the global burden of young infant sepsis.

\section{MEDICATION ERRORS AND NEAR-MISSES IN A PEDIATRIC EMERGENCY DEPARTMENT: A RETROSPECTIVE REVIEW}

Ann Loh, Sung Shin Teng, Khai Pin Lee, Sashikumar Ganapathy. Singapore

\subsection{6/bmjpo-2021-RCPCH.213}

Background Medication errors (MEs) are a significant cause of preventable morbidity and mortality. The paediatric emergency department (ED) is a high-risk setting with high patient volume and acuity of care, serving a uniquely susceptible population where weight-based calculations render them vulnerable to dosing errors. Medications are also often kept in stock, and are not audited by a pharmacist prior to administration. Stress, noise, time pressures, and unfamiliarity with paediatric conditions amongst rotating trainees compound this risk.

Objectives To describe the occurrence and type of MEs in pediatric ED and to identify contributing factors.

Methods A retrospective review of all reported MEs in Singapore's largest tertiary pediatric ED from January 2013 to December 2019. MEs were reported via Risk Management System (RMS), while near-misses were extracted from RMS and the pharmacy department's Closed Loop Medication Management System. Descriptive statistics were used to present ME types, severity and contributing factors.

Results Of 101 MEs reported in RMS, 59\% were related to wrong dose, $22 \%$ to wrong medication and $6 \%$ to wrong patient. Wrong doses were related to duplicate dose $(48 \%)$, wrong weight (20\%), 10-fold errors (5\%) and calculation errors (5\%). Majority of MEs occurred during drug 
administration (52\%), followed by prescription (30\%) and transcription of medications (12\%). Most commonly involved medications in MEs were anti-pyretics (33\%), those for respiratory conditions $(15 \%)$ and analgesics (13\%). Of the MEs, 9 (9\%) were serious errors requiring enhanced monitoring and/ or intervention, while $1(1 \%)$ caused temporary patient harm. Human factors (92\%) was the most frequently documented contributing factor, namely failure to comply with established workflows, accurate information provided by guardians, and distractions.

Conclusions We have described a low incidence of MEs from 2013 to 2019. Strategies should be developed to ameliorate MEs in the pediatric ED. Areas for focused intervention should include systematic evaluation of drug administration and prescription processes, to enhance compliance with established procedures of checking (amongst the healthcare team and with guardians) and putting safeguards in place to minimize the impact of distractions in the ED setting.

\section{PROMOTING EARLY INTERVENTION FOR SPECIAL- NEEDS CHILDREN AS A BRANCH SERVICE OF PEDIATRIC NEUROLOGY}

Pyae Phyo Aung, Nay Linn, Kyaw Linn, Aye-Mya Min-Aye, Ayemu Saan, Nway Nway. Myanmar

\subsection{6/bmjpo-2021-RCPCH.214}

Background Since the establishment of pediatric neurology services in 2011, pediatric neurology team has been dealing with increasing numbers of different neurological problems year by year. Developmental, speech and language and behavioral problems are quite common in out-patient clinics. In 2017, UNICEF estimated that $42 \%$ to $45 \%$ of young Myanmar children need early childhood intervention services. So Child Development Center (CDC) was launched in 2017 as a branch of Pediatric Neurology with services such as developmental screening, assessment and appropriate intervention.

Objectives The objective of the study is to evaluate early intervention services for children who were enrolled in CDC.

Methods Retrospective study was done on early intervention services in CDC through the review of CDC registration records from 2017 to 2020. The study population included all the children referred for screening, assessment and interventions. After enrollment, thorough history, and relevant general, systemic as well as neurological examinations and necessary investigations were performed for diagnosis and underlying causes. Pediatric neurologists also conducted developmental screening and necessary neurodevelopmental assessments by using instruments such as Schedule of Growing Skills-2, Conner Rating Scale and DSM-5 Diagnostic Criteria. Then, appropriate interventions such as physiotherapy, occupational, speech, behavior and self-care therapies were given by allied professionals in voluntary participation and paraprofessionals. The extracted data from CDC registry to analyze were demographic variables, presenting symptoms, medical history and examination findings, results of developmental assessment and diagnoses and therapy services.

Results A total of 138 children with girls (30.43\%) and boys $(69.57 \%)$ were enrolled. The youngest age at enrollment was 1 year, the oldest 15 year with a mean age of 4.558 year. The most common developmental problems were global developmental delay (GDD) (54.35\%), autism spectrum disorder

\begin{tabular}{|c|c|c|c|c|}
\hline \multirow{2}{*}{$\begin{array}{l}\text { Demographic } \\
\text { characteristics }\end{array}$} & \multicolumn{4}{|c|}{ Total $\mathrm{N}=138$} \\
\hline & $n$ & $\%$ & Mean & SD \\
\hline Gender & 42 & 30.43 & & \\
\hline Female & 96 & 69.57 & & \\
\hline \multicolumn{5}{|l|}{ Male } \\
\hline Age (Year) at enrollment & & & 4.558 & 2.628 \\
\hline \multicolumn{5}{|l|}{ **minimum age is 1 year } \\
\hline Age (Year) & & 72.46 & & \\
\hline $0-5$ & 100 & 22.46 & & \\
\hline $6-10$ & 31 & 5.07 & & \\
\hline $11-15$ & 7 & & & \\
\hline Age (Year) of GDD & & & 3.259 & 1.231 \\
\hline \multicolumn{5}{|l|}{ diagnosis } \\
\hline \multicolumn{5}{|l|}{${ }^{* *}$ minimum age is 1 year } \\
\hline Age (Year) of ASD & & & 3.75 & 1.939 \\
\hline \multicolumn{5}{|l|}{ diagnosis } \\
\hline \multicolumn{5}{|l|}{${ }^{* *}$ minimum age is 2 years } \\
\hline 2017 Assessment & 23 & 16.67 & & \\
\hline 2018 Assessment & 51 & 36.96 & & \\
\hline 2019 Assessment & 51 & 36.96 & & \\
\hline 2020 Assessment & 13 & 9.42 & & \\
\hline
\end{tabular}

(ASD) (17.39\%) and speech and language delay (SLD) (16.67\%) with male-predominance in GDD (64.81\%), ASD $(79.17 \%)$ and SLD (78.26\%) respectively. Speech problems (97.62\%), language (97.5\%) and adaptive skills (93.33\%) delay and behavior problems (37.93\%) were the most common presentation in GDD. Youngest age for ASD by DSM-5 was 2 years with a mean age of 3.75 years. Problem-in-makingfriends (75\%), poor-eye-contact $(70.83 \%)$, stereotypies $(54.17 \%)$ and odd-play (41.67\%) were the most encountered autism symptoms in children with ASD. Rising trend was found in assessment (36.96\% in both 2018 and 2019) and therapy services in comparison with 2017.

Conclusions This study explores the promotion of early intervention services for special-needs children as part of pediatric neurology. Scaling up better holistic intervention services in multidisciplinary approach and the study aiming for the outcome of children benefiting from CDC services are further considerations against impact of COVID-19 pandemic in 2020 .

\section{CAPTURING THE RESPONSE TO THE COVID-19 PANDEMIC IN A UK DISTRICT GENERAL HOSPITAL PAEDIATRIC DEPARTMENT}

Felicity Cooksey, Anna Cripps, Gita Modgil. UK

\subsection{6/bmjpo-2021-RCPCH.215}

Background In response to the coronavirus pandemic, the paediatric team have needed to make rapid changes to our pathways and ways of working. In this time colleagues have fast tracked the testing of new processes and generated ideas to deliver continuous improvement.

Objectives General objective: To capture the rapid changes made to healthcare practices within the Paediatric department at Musgrove Park Hospital, Taunton in response to the coronavirus pandemic and to share learning across the directorate. 